\title{
Management of Vestibular Schwannoma: Dependence on Stakeholder's View for Small and Medium-Sized Tumors
}

\author{
Haralampos Gouveris, Maria Zisiopoulou, Wolf J Mann \\ Department of Otorhinolaryngology, The University of Mainz Hospitals and Clinics, Mainz, Germany
}

Correspondence: Haralampos Gouveris, Department of Otorhinolaryngology, The University of Mainz Hospitals and Clinics Langenbeckstr 1, 55131 Mainz, Germany, Phone: +49-6131-177361, Fax: +49-6131-176637, e-mail: hagouve@yahoo.de

\begin{abstract}
Management options for patients with vestibular schwannoma include observation and active treatment, namely surgical resection, gamma knife stereotactic radiosurgery or fractionated radiation therapy. Although for large tumors microsurgery remains the mainstay of treatment, management of the small- and medium-sized vestibular schwannomas has been the matter of considerable controversy. Computerized clinical decision support systems have been developed to assist clinicians in this demanding task. Nonetheless, the complexity of the problem requires adaptation of the decision strategy to specific circumstances arising within a particular clinical scenario which can not always be modeled with adequate precision or addressed adequately within a mathematical framework. We present a set of clinical, neurophysiologic and radiologic parameters and the respective evidence which may guide carers' decisions. These parameters include the size, growth rate and localization of the tumor, the age, general medical condition, facial nerve function, hearing and hearing deterioration rate, balance, vestibular and trigeminal nerve function of the affected individual, the estimated risk of malignant transformation and radiation-induced tumors, the health-related quality of life measures, the patient's and surgeon's preference and the issue of cost-effectiveness. A complex decision analysis, guided by evidence and tailored to each individual patient is required.
\end{abstract}

Keywords: Decision, Evidence, Management, Observation, Radiation, Surgery, Schwannoma, Vestibular.

\section{INTRODUCTION}

$M$ anagement options for patients with vestibular schwannoma include observation (al so referred to as conservative management) and the so-called "active" treatment, which includes the modalities of surgical resection, gamma knife stereotactic radiosurgery (GK-SRS) and fractionated radiation therapy (FRT).

Although, for large tumors microsurgery remains the mainstay of treatment, management of thesmall-and mediumsized vestibular schwannomas has been the matter of considerable controversy. Computerized clinical decision support systems have al ready been developed to assist clinicians in this demanding task. N onethel ess, there is little doubt about the fact that the complexity of the problem requires adaptation of the decision strategy to specific circumstances arising within a particular clinical scenario, which cannot al ways be modeled with adequate precision or addressed adequately within a mathematical framework. H owever, a set of general qualitative rules may guide carers' decisions. To shed more light into the anatomy of this cognitivedecision process, onemay try to dissect theseparate factors affecting the final management decisions and decisions should be guided by existing evidence on these factors.

\section{SIZE AND GROWTH RATE OF THE TUMOR}

Tumor size is measured by magnetic resonance imaging (MRI). For ease of communication of findings between clinicians and researchers, it is generally advisable to report $M R I$ results according to the guidelines advanced by the Committee on Hearing and Equilibrium of the A merican A cademy of O tolaryngology Head and Neck Surgery Inc. $(1995)^{42}$ or the guidelines elaborated by the Tokyo consensus meeting. ${ }^{1}$ In the latter case, estimation of the size of extrameatal tumors involves measuring the largest extrameatal diameter on axial MR images; intrameatal tumors are measured parallel to the internal auditory canal (IAC). A ccording to the A merican A cademy guidelines, only the extrameatal (extracanalicular) portion of the tumor should be measured and two linear measurements should be made on the axial M RI image showing the largest tumor size; the diameter of the tumor in the direction parallel to the petrous ridge and the maximum diameter of the tumor in an orientation perpendicular to the first diameter. Tumors limited to the internal auditory canal should be reported separately as 'intracanalicular' tumors and their length within the IAC may be recorded at the discretion of the investigator.

Some authors advocate that the first M R imaging study should be performed a year after diagnosis, and subsequent imaging should be performed yearly or every 2 years depending on the appearance of new symptoms, tumor growth or both. ${ }^{2}$ Nonetheless, for patients managed conservatively, other authors recommend an initial magnetic resonance imaging scan at 6 months with scans to take place 
at annual intervals for 2 years. A further scan 2 years later will identify any patient with indolent tumors. Thereafter, follow-up should be life-long every 5 years. Cystic tumors represent a particular threat to patients and should only be treated conservatively with caution. ${ }^{3}$

In retrospective review of patients with tumors that demonstrated growth of greater than $1 \mathrm{~mm} /$ year between two consecutive scans, Mick et al $(2009)^{4}$ found that in $63.9 \%$ of the patients the tumors continued to grow, $30.6 \%$ stayed the same size and $5.6 \%$ regressed in size. Of note, the average follow-up period after growth was identified to be of 2.1 years. In a retrospective review of patients with conservatively managed $\mathrm{VS}$, the mean tumor grow th rate at the 1-year follow-up was significantly higher in the group requiring treatment $(3.0 \mathrm{~mm})$ than in the group not requiring treatment $(0.36 \mathrm{~mm})$. Thus, the tumor growth rate at the 1-year follow-up was a strong predictor of the eventual need for treatment. ${ }^{5}$

Patients with purely intracanalicular tumors managed with observation had a 5-year no-growth rate of $89.8 \%$ compared with 73.9 and $45.2 \%$ for Grade I and G rade II or larger tumors respectively. The difference between intracanalicular and Grade II or larger tumors was statistically significant. ${ }^{6}$ The overall 5 -year no-growth rate recorded in the patients studied by Solares and Panizza (2008) who were managed conservatively, was $70.6 \%$ and in patients with growth the 5-year no-intervention rate was $81.3 \%$. Additionally, $10 \%$ of these patients demonstrated tumor regression. These authors suggested that treatment can be delayed in a large proportion of vestibular schwannoma patients, particularly in patients with small tumors, and recommended a period of observation to determine the need for treatment in patients without indications for urgent intervention.

A growth rate of $>2.5 \mathrm{~mm} /$ year was a better predictor of hearing loss than the initial tumor size for patients undergoing observation management of vestibular schwannomas smaller than $25 \mathrm{~mm}$ in largest diameter. ${ }^{7}$

In patients who were followed conservatively and who exhibited a 1-year tumor growth rate equal to or greater than $3 \mathrm{~mm}$, conservative treatment was often discontinued. ${ }^{2}$ Growth rates of intrameatal $(1.02 \pm 1.8 \mathrm{~mm} / \mathrm{year})$ and extrameatal ( $1.40 \pm 3.1 \mathrm{~mm} /$ year) tumors did not differ significantly. No significant association was found among tumor growth rate and sex, age, initial hearing status or initial tumor grade. Delay in diagnosis was the only significant factor associated with tumor grow th rate. ${ }^{2}$

Patients with intracanalicular and small/medium-sized tumors have been followed prospectively by Ferri et al (2008). A Imost two-thirds (64.5\%) of the cases did not show tumor growth during the entire observation period (mean follow-up period, 4.8 years). A mong growing tumors, patients that were surgically treated showed no complications or facial nerve palsy.
Enlargement of at least $2 \mathrm{~mm}$ after radiosurgery may occur in $14 \%$ of treated patients after a median follow-up of 56 months (Pollock, 2006). A mong the 28 patients who did not undergo resection at the time of initial enlargement, three patterns were identified on later imaging. Sixteen (57\%) patients showed eventual tumor regression (type 1), and eight (29\%) patients had tumors that increased and remained larger but did not show progressive enlargement (type 2). Four (14\%) patients showed progressive enlargement on serial imaging (type 3) and underwent additional treatment. As a result, tumor expansion after V S radiosurgery rarely denotes a failed procedure, and the majority of patients only require further imaging. A pproximately, one-third of tumors that enlarge will remain increased in size compared with the time of radiosurgery but will not show sequential growth. Additional tumor treatment should be reserved only for patients who demonstrate progressive tumor enlargement on serial imaging. ${ }^{8}$

\section{LOCALIZATION}

Purely intracanalicular tumors pose specific challenges in their management. Niranjan et al $(2008)^{9}$ reported preservation of serviceable hearing in 31 of 40 (77.5\%) patients treated by gamma knife stereotactic radiosurgery (GK-SRS) who had initial A merican A cademy of O tolaryngology - Head and N eck Surgery Class A hearing. Serviceable hearing was preserved in 40 of 79 (64.5\%) patients with Gardner-Robertson (GR) Grade I or II preGK -SRS hearing. Hearing grades improved in seven patients. Facial and trigeminal nerve function was preserved in all patients. The tumor control rate (freedom from additional intervention) was $99.0 \%$ (95 of 96 ) at a median follow-up of 28 months (range was 12-144 months).

Hajioff et al $(2008)^{10}$ followed prospectively by serial clinical examination, M RI scans and audiometry, 72 patients with a unilateral vestibular schwannoma that have been treated conservatively for a median of 121 months. Cerebellopontine angle tumors grew faster $(1.4 \mathrm{~mm} /$ year $)$ than intracanalicular tumors $(0 \mathrm{~mm} / \mathrm{year}, \mathrm{p}<0.01)$.

Solares and Panizza (2008) found that patients with purely intracanalicular tumors managed with observation had a 5-year no-grow th rate of $89.8 \%$ (compared with $73.9 \%$ and $45.2 \%$ for Grade I and Grade II or larger tumors respectively).

\section{AGE AND GENERAL MEDICAL CONDITION}

Patients, 65 years or older, at time of diagnosis with smaller tumors $(<2.5 \mathrm{~cm})$ were followed with serial magnetic resonance imaging by Roehm and Gantz (2007). ${ }^{11}$ If significant growth occurred, then they were treated with surgery (average growth in the surgical group was $4.1 \mathrm{~mm} /$ 
year vs $0.3 \mathrm{~mm} /$ year for patients remaining in the observation group). Surgery was performed at initial diagnosis on patients with larger tumors or in selected patients for hearing preservation. Stereotactic radiosurgery was performed for poor surgical candidates and for patient choice. Perry et al $(2001)^{12}$ followed patients over the age of 65 years without prior treatment or observation with serial, gadoliniumenhanced M RI scans performed at 6 months and then yearly, if no significant growth had occurred. The patients were followed for an average of 3.5 years. The average tumor size at presentation was $1.14 \mathrm{~cm}$, with a range of growth rates from 0 to $1.2 \mathrm{~cm}$ per year. Only $12 \%$ of the patients required further intervention. No patients developed significant complications during the observation period. These authors ${ }^{12}$ concluded that vestibular schwannomas in the older population can be managed safely using serial M RI scanning. No correlation could be made between initial tumor size and subsequent growth rate in this patients' group.

In younger patients, resection is often advocated because of concern regarding the long-term effects of radiation. L obato-Polo et al $(2009)^{13}$ review ed long-term outcomes in 55 patients with vestibular schwannomas 40 years of age or younger, who underwent GK-SRS and were followed up for a minimum of 4 years. The 5 -year rate of freedom from additional management was $96 \%$. No patient developed a secondary radiation-related tumor.

In a retrospective analysis of 20 patients under 21 years of age who underwent surgery via the retrosigmoid approach, the statistical comparison of the postoperative status including cochlear and facial nerve function, and complications did not show any significant difference between young and adult patients. ${ }^{14}$

\section{Facial Nerve Function}

A n overall facial nerve preservation rate of $96.2 \%$ was found after gamma knife stereotactic radiosurgery for vestibular schwannoma in the cumulative analysis of 23 published studies performed by $Y$ ang et al (2009). ${ }^{15}$ Patients receiving less than or equal to $13 \mathrm{G}$ y of radiation at the marginal dose had a better facial nerve preservation rate than those who received higher doses. Patients with a tumor volume less than or equal to $1.5 \mathrm{~cm}^{3}$ also had a greater facial nerve preservation rate than patients with tumors greater than $1.5 \mathrm{~cm}^{3}$. Superior facial nerve preservation was also noted in patients younger than or equal to 60 years of age.

None of the patients treated by GK -SRS with doses lower than 13 Gy experienced facial or trigeminal neuropathy. ${ }^{13}$ Facial nerve function was preserved in $98.2 \%$ of patients.

There is no doubt that the principal concerns about facial nerve function involve the motor component of the nerve.
There are fewer data on the impact of dysfunction of the sensory component of the facial nerve (intermediate nerve) with the accompanying taste, nasal secretory and lacrimal disorders both at presentation as well as after treatment, findings that may be present in more than $50 \%$ of patients treated by surgery. ${ }^{16}$

\section{Hearing (and Hearing Deterioration Rate)}

Monitoring hearing has been used quite frequently as a clinical marker of $\mathrm{VS}$ progression. A growth rate of $>2.5 \mathrm{~mm} / \mathrm{year}$ is a better predictor of hearing loss than the initial tumor size for patients undergoing observation management of vestibular schwannomas $<25 \mathrm{~mm}$ in largest diameter. ${ }^{7}$ These authors had excluded in their study patients with poorer hearing (A merican A ssociation of Otolaryngology - Head and Neck Surgery Classes C or D, or Gardner-R obertson Classes III, IV or V) at the time of presentation. In young patients treated with GK-SRS, hearing preservation rates (i.e. remaining within the same Gardner-Robertson hearing class) were 93,87 and $87 \%$ at 3, 5 and 10 years respectively. M ore specifically, in patients with serviceable hearing before SRS, hearing was maintained in 100, 93 and $93 \%$ of patients at 3,5 and 10 years respectively. Hearing preservation was related to a margin dose lower than $13 \mathrm{~Gy} .{ }^{13} \mathrm{~A}$ t presentation, $45.5 \%$ of the patients with intracanalicular and small/medium-sized tumors who have been followed prospectively by Ferri et al $(2008)^{17}$ had useful hearing (classes $A$ and $B$ of the A merican A cademy of Otolaryngology- Head and Neck Surgery classification), and $41(73.2 \%)$ patients had preserved hearing during follow-up independently from the tumor growth rate. The mean follow-up period in the study by Ferri et al (2008) ${ }^{17}$ was 4.8 years. In the cohort of patients treated conservatively by Hajioff et al $(2008)^{10}$ for a median of 121 months, hearing deteriorated substantially even in tumors that did not grow, but did so faster in tumors that grew significantly. M ean deterioration in pure tone average at $0.5,1,2$ and $3 \mathrm{kHz}$ was $36 \mathrm{~dB}$ and speech discrimination scores deteriorated by $40 \%$.

\section{Balance and Vestibular Function}

V ertigo, dizziness and disorders of body posture are present in $52 \%$ of untreated VS patients. ${ }^{18} \mathrm{U}$ sing the Short Form36 and the Glasgow Benefit Inventory, Myrseth et al $(2006)^{19}$ found that vertigo is the symptom causing the most pronounced negative effect on quality of life $(Q \circ L)$ in patients with vestibular schwannoma. Surgical treatment should be considered in patients with small- or mediumsized tumors and persisting disabling vertigo resulting in a poor quality of life. Vestibular schwannoma patients with disabling vertigo, experience significant reduced quality of 
life when compared with a healthy population. In this specific group of patients, translabyrinthine tumor removal significantly improved the patients' quality of life. ${ }^{20}$ In their prospective study, these authors found a significant improvement of Dizziness Handicap Inventory (DHI) total scores and Short Form-36 scales on physical and social functioning, role-physical functioning, role-emotional functioning, mental health and general health at 12 months after surgery when compared with preoperative scores. The size of an inferior vestibular nerve (IVN) and vestibular schwannoma (VS) correlated with computerized dynamic platform posturography findings, suggest that the nerve branch of origin of a VS appears to influence the nature of vestibular adaptation mechanisms in untreated V S tumors. ${ }^{21}$ In IV N vestibular schwannoma patients the visual system plays a major role in the maintenance of postural stability. Postoperatively, accelerated vestibular compensation was achieved in patients who underwent surgery by means of a middle fossa or retrosigmoid approach with anatomical preservation of one branch of the vestibular nerve. ${ }^{22}$ Three months after surgery, $47 \%$ of the latter patients were back to work without substantial restrictions compared with $29 \%$ of the group of patients who had undergone surgery by a translabyrinthine approach. Nonetheless, at the end of the sixth postoperative month there was no significant difference between these two groups. ${ }^{22}$

\section{Trigeminal Nerve Function}

A lthough, an issue that attracts in general little attention, functional disorders of the trigeminal nerve may be quite important from the patient's perspective.

None of the patients treated with GK-SRS with doses lower than $13 \mathrm{~Gy}$ experienced facial or trigeminal neuropathy. ${ }^{13}$ These authors reported that trigeminal nerve function was preserved in $96.4 \%$ of their patients.

In a study comparing GK - SRS to microsurgical resection by means of translabyrinthine, subocci pital and middl e fossa approaches, Karpinos et al (2002) ${ }^{23}$ found that the rate of trigeminal neuropathy was significantly higher in the microsurgical group than in the GK -radiosurgical group ( $17 \%$ vs $0 \%$ in the immediate postoperative period, $p<001$, and $22 \%$ vs $12.2 \%, p=0.009$, at long-term follow-up).

\section{RISK OF MALIGNANT TRANSFORMATION AND RADIATION-INDUCED TUMORS}

$M$ alignant transformation of sporadic vestibular schwannoma has been reported after GK -SRS therapy. ${ }^{24}$ $M$ al ignant transformation may occur even 8 years after $G K$ SRS. ${ }^{25}$ The overall risks of tumor induction from radiotherapy in people without tumor-predisposing conditions appear to be quite small, but this is not the case for people with tumor-predisposing conditions. ${ }^{26} \mathrm{~A} n$ al most seven-fold increased risk of malignancy may exist for irradiated as compared to nonirradiated neurofibromatosis 2 (NF2) patients. ${ }^{27}$ A pproximately $5 \%$ of all vestibular schwannomas occur in NF2 patients, but half the reported malignant transformations occur in NF2 patients. ${ }^{28} \mathrm{M}$ uch more caution is warranted regarding the use of radiation treatment for benign tumors in childhood and in tumor-prone conditions, such as the neurofibromatoses. ${ }^{26} \mathrm{H}$ asegawa et al $(2005)^{29}$ reported on their series of sporadic VS patients treated by GK-SRS with a follow-up period more than 10 years (median duration of follow-up was 135 months). No patient showed malignant transformation in their series. A dditionally, malignant transformation may occur many years after fractionated radiotherapy (FRT), so caution is advocated when using this treatment for young patients. $M$ aire et al (2006) ${ }^{30}$ reported a case of malignant transformation of a VS 216 months after initial FRT treatment.

A malignant peripheral nerve sheath tumor arising from a benign schwannoma of the eighth cranial nerve has been reported in the literature. Initially, the tumor had been surgically debulked. On surgical revision, malignant areas were discovered within the benign tumor, which were considered to represent a malignant transformation of a previously benign tumor. ${ }^{31}$ Given the extremely low rate of malignant transformation in irradiated sporadic VS, one cannot exclude the possibility of spontaneous malignant transformation of V S tumors, as illustrated in the latter case.

The risk of radiation-induced tumors after radiosurgery is unknown. Radiation-induced neoplasms are extremely rare after stereotactic radiosurgery. ${ }^{32}$ In a recent study of 55 patients aged 13 to 40 years treated by GK-SRS after a follow-up period ranging between 4 and 20 years (mean: 5.3 years) no patient developed a secondary radiation-related tumor. ${ }^{13}$

\section{HEALTH-RELATED QUALITY OF LIFE (QOL) MEASURES}

In the study by $\mathrm{V}$ ogel et al (2008) ${ }^{33}$ the 36 -Item Short Form health survey scores of VS patients at diagnosis were significantly decreased when compared with healthy controls, patients with head and neck cancer, benign prostate hypertrophy, chronic obstructive pulmonary disease and deaf patients. Scores for ill ness perceptions were in between those of patients with acute pain and chronic pain for most subscales. Changing illness perceptions and coping by means of an intervention and encouraging social support by means of patient support groups may improve QoL in VS patients. ${ }^{33}$ 
Return-to-work should become a standard reporting criterion for postoperative Q o L in V S patients. ${ }^{34}$ In the study by Lobato-Polo et al (2009), ${ }^{13}$ all patients continued their previous level of activity or employment after GK -SRS.

N onethel ess, a major methodological flaw of studies on heal th-related Q $\mathrm{oL}$ in V S patients is that they do not include in their design a prospective comparison between $\mathrm{QoL}$ measures before and after any form of treatment in their patient cohorts. 35

In their analysis, Whitmore et al $(2011)^{36}$ found that at 5 years, patients treated with radiosurgery have an overall better Q $\mathrm{LL}$ than those treated with either microsurgery or those investigated further with serial imaging. The authors found that the complications associated with wait-and-scan and microsurgery treatment strategies negatively impacted patient lives more than the complications from radiosurgery. One limitation of this study is that the 10-year follow-up data were too limited to analyze, and more studies are needed to determine if the authors' results will still be consistent at 10 years.

\section{PATIENT'S AND SURGEON'S PREFERENCE}

In a study by Cheung et al (2010), ${ }^{37}$ all stakeholder groups (younger and older prospective patients, observation patients and surgeons) indicated that GK -SRS radi osurgery was their first choice among realistic treatments. $Y$ ounger and older prospective patients had indistinguishable first-choice profiles. Tumor surgeons had a higher preference for hearing destructive translabyrinthine craniotomy compared with younger and older prospective observation patients. This finding, al though seemingly surprising, is entirely consistent with the group's higher importance scores for both temporary facial nerve weakness and long-term cancer risks. Tumor surgeons are relatively risk averse to facial disfigurement and potentially mortal disease. ${ }^{37}$ In the same study, younger and older prospective patients had indistinguishable preference profiles, implying that age should not be used as a factor to eliminate certain treatment choices from consideration.

\section{COST-EFFECTIVENESS}

In their study involving patients treated with microsurgery or GK -SRS at the M ayo Clinic (M innesota, USA ), B anerjee et al $(2008)^{38}$ found that although the total cost of microsurgery is higher due to the costs of hospitalization, follow-up costs for radiosurgery are greater in general. From a societal perspective, radiosurgery was less expensive than microsurgical resection provided that the rate of tumor progression after radiosurgery remains low with long-term follow-up. Cost analysis within the Canadian health care system ${ }^{39}$ determined the mean total cost per patient for microsurgical removal at CA D $\$ 22,402$ ( $£ 12,545 ; 14,561 €)$, for gamma knife radiotherapy at CAD $\$ 27,659$ ( $£ 15,489$;
$17,978 €)$, for LINAC fractionated radiation therapy at CAD $\$ 9,003(£ 5,041 ; 5,852 €)$ and for conservative management at CAD $\$ 9,651(£ 5,405 ; 6,273 €)$ over the follow-up timeframe. The authors concluded that an economic advantage could be demonstrated for the conservative management of vestibular schwannomas compared to microsurgical removal and gamma knife radiotherapy on the proviso that no increase in active treatment complications arose from continued tumor growth during the period of observation. ${ }^{39} \mathrm{~N}$ onetheless, as the some authors suggested, cost conclusions should be applied cautiously to individual and wider societal decisions. Important reasons for this fact are the infrastructural costs covered by a public health care system, the indirect and intangible costs, such as time off work, psychological impact, caregiver costs as well as pain and suffering, which were not considered in their study. ${ }^{39}$ It was also acknowledged that cost studies are very sensitive to the nation and system within which the study was performed and that various public or private health care systems can have widely discrepant variations in hospital costs and charges.

\section{DECISION ANALYSIS}

A computerized clinical decision analysis was undertaken by Telian $(1994)^{40}$ to model the decision to observe small vestibular schwannomas or proceed to immediate surgery. The decision tree's construction accounted for tumor size, hearing level, and other critical variables, such as the individual's aversion to unilateral hearing loss and facial paralysis. Unless life expectancy was short, the analysis suggested that surgery at the time of diagnosis was appropriate, assuming that growth of the tumor was anticipated. V ariations in surgical proficiency and patient risk aversion within expected ranges did not influence the decision to operate. The most critical variable was the probability that the tumor would remain stable in size. As mentioned above, Cheung et al $(2010)^{37}$ used conjoint analysis to study prospectively treatment preference profiles among vestibular schwannoma stakeholders, namely younger and older prospective patients, observation patients and neurotologic surgeons. Stakeholders were assessed for the synthetic VS case scenario of a $10 \mathrm{~mm}$ tumor in association with useful hearing and normal facial function. They found some notable distinctions among the aforementioned groups: (1) Permanent deafness was less important to tumor surgeons (than to all other patient groups), (2) temporary facial weakness was more important to tumor surgeons and observation patients, and (3) cancer in the long-term was less important to observation patients. Conjoint analysis is advocated by Cheung et al $(2010)^{37}$ as 
a promising tool to empower patient self-directed analysis of treatment preference profiles. Patients will expectedly benefit from transparency conferred by an impartial decision tool and become highly informed partners in shared medical decision making.

Using Markov decision analysis from the societal perspective and the benefit derived from each management strategy in quality-adjusted life years (QALY s) as the main outcome measure, M orrison (2010) ${ }^{41}$ found that for patients with small $(<1.5 \mathrm{~cm})$ vestibular schwannomas of all ages, a period of observation during which tumor growth and hearing thresholds are closely monitored was the superior strategy. For tumors that grow substantially or when hearing deteriorates, definitive management via radiosurgery was recommended.

As Cheung et al $(2010)^{37}$ put it, decision models cannot fully capture vagaries of human emotion under duress, multitude of medical conditions that impact on decision making, and accumulated experiential perspectives. There is no substitute for consultation with a seasoned provider to help the patient place her or his treatment preferences for a specific disease in the context of comorbid conditions.

\section{REFERENCES}

1. Kanzaki J, Tos M, Sanna M, M offat DA, M onsell EM, Berliner KI. New and modified reporting systems from the consensus meeting on systems for reporting results in vestibular schwannoma. Otol N eurotol 2003;24:642-48.

2. Bakkouri WE, Kania RE, Guichard JP, L ot G, Herman P, Huy PT. Conservative management of 386 cases of unilateral vestibular schwannoma: Tumor growth and consequences for treatment. J N eurosurg 2009;110:662-69.

3. M artin TP, Senthil L, Chavda SV, W alsh R, Irving RM . A protocol for the conservative management of vestibular schwannomas. Otol Neurotol 2009;30:381-85.

4. M ick $P, W$ esterberg $B D, N$ go R, A kagami R. Growing vestibular schwannomas: What happens next? Otol Neurotol 2009;30: 101-04.

5. Deen HG, Ebersold MJ, Harner SG, B eatty CW, M arion M S, W haren RE, G reen JD, Q uast L. C onservative management of acoustic neuroma: A n outcome study. Neurosurgery 1996;39:260-64.

6. Solares CA, Panizza B. V estibular schwannoma: An understanding of growth should influence management decisions. Otol Neurotol 2008;29:829-34.

7. Sughrue ME, Y ang I, A randa D, Lobo K, Pitts LH, Cheung SW, Parsa AT. The natural history of untreated sporadic vestibular schwannomas: A comprehensive review of hearing outcomes. J N eurosurg 2010;112:163-67.

8. Pollock BE. M anagement of vestibular schwannomas that enlarge after stereotactic radiosurgery: Treatment recommendations based on a 15-year experience. Neurosurgery 2006; 58:241-48.

9. Niranjan A, M athieu D, Flickinger J C, K ondzi olka D, L unsford LD. Hearing preservation after intracanalicular vestibular schwannoma radiosurgery. N eurosurgery 2008;63:1054-62.
10. Hajioff $D$, Raut VV, W alsh RM, B ath A P, Bance M L, Guha A, Tator $\mathrm{CH}$, Rutka JA. Conservative management of vestibular schwannomas: Third review of a 10-year prospective study. C lin Otolaryngol 2008;33:255-59.

11. Roehm PC, Gantz BJ. M anagement of acoustic neuromas in patients 65 years or older. Otol Neurotol 2007;28:708-14.

12. Perry BP, Gantz BJ, Rubinstein JT. A coustic neuromas in the elderly. Otol Neurotol 2001;22:389-91.

13. Lobato-Polo J, K ondziolka D, Zorro O, K ano H, Flickinger JC, L unsford LD. Gamma knife radiosurgery in younger patients with vestibular schwannomas. N eurosurgery 2009;65:294-300.

14. M irzayan MJ, Gerganov V M, Lüdemann W, Oi S, Samii M, Samii A. M anagement of vestibular schwannomas in young patients: Comparison of clinical features and outcome with adult patients. Childs Nerv Syst 2007;23:891-95.

15. $Y$ ang I, Sughrue M E, H an SJ, Fang S, A randa D, Cheung SW, Pitts LH, Parsa AT. Facial nerve preservation after vestibular schwannoma gamma knife radiosurgery. J Neurooncol 2009;93:41-48.

16. Stripf T, B raun K, Gouveris H, Stripf EA, M ann WJ, A medee RG. Influence of different approaches to the cerebellopontine angle on the function of the intermediate nerve. J Neurosurg 2007;107:927-31.

17. Ferri GG, M odugno GC, Pirodda A, Fioravanti A, Cal bucci F, Ceroni AR. Conservative management of vestibular schwannomas: An effective strategy. Laryngoscope 2008; 118:951-57.

18. Gouveris H, Stripf $T$, Victor A, Mann W. Dynamic posturography findings predict balance status in vestibular schwannoma patients. Otol Neurotol 2007;28:372-75.

19. M yrseth E, Møller P, W entzel-Larsen T, Goplen F, L undJ ohansen M. Untreated vestibular schwannomas: V ertigo is a powerful predictor for heal th-related quality of life. N eurosurgery 2006;59:67-76.

20. Godefroy W P, Hastan D, van der M ey A G. Translabyrinthine surgery for disabling vertigo in vestibular schwannoma patients. Clin Otolaryngol 2007;32:167-72.

21. Gouveris H, A kkafa S, Lippold R, M ann W. Influence of nerve of origin and tumor size of vestibular schwannoma on dynamic posturography findings. A cta Otolaryngol 2006;126:1281-85.

22. M aurer J, Frommeld T, M ann W. V estibular function after acoustic neuroma removal with preservation of one branch of the vestibular nerve. Otol Neurotol 2002;23:749-54.

23. K arpinos $M$, T eh BS, Zeck $O$, Carpenter LS, Phan C, M ai WY . Treatment of acoustic neuroma: Stereotactic radiosurgery vs. microsurgery. Int J Radiat Oncol Biol Phys 2002;54:1410-21.

24. Shin M, U eki K, K urita H, K irino T. M alignant transformation of a vestibular schwannoma after gamma knife radiosurgery. L ancet 2002;360:309-10.

25. A kamatsu $Y, M$ urakami $K, W$ atanabe $M$, J okura $H$, Tominaga $T$. $M$ alignant peripheral nerve sheath tumor arising from benign vestibular schwannoma treated by gamma knife radiosurgery after two previous surgeries: A case report with surgical and pathological observations. World Neurosurg 2010;73:751-54.

26. Evans DG, Birch JM, Ramsden RT, Sharif $S$, Baser ME. $M$ alignant transformation and new primary tumours after therapeutic radiation for benign disease: Substantial risks in certain tumour prone syndromes. J M ed Genet 2006;43: 289-94.

27. Baser M E, Evans D GR, J ackler RK, Sujansky E, Rubinstein A. $M$ alignant peripheral nerve sheath tumours, radiotherapy and neurofibromatosis 2. B r J Cancer 2000;82:998. 
28. Thomsen J, Mirz F, W etke R, A strup J, Bojsen-M oller M, $\mathrm{Niel}$ sen $\mathrm{E}$. Intracranial sarcoma in a patient with neurofibromatosis type 2 treated with gamma knife radiosurgery for vestibular schwannoma. A m J Otol 2000;21:364-70.

29. Hasegawa $T, K$ ida $Y, K$ obayashi $T, Y$ oshimoto $M, M$ ori $Y$, Y oshida J. L ong-term outcomes in patients with vestibular schwannomas treated using gamma knife surgery: 10-year follow-up. J Neurosurg 2005;102:10-16.

30. Maire JP, Huchet A, M ilbeo $Y$, Darrouzet $V$, Causse $N$, Célérier $D, L$ iguoro $D, B$ ébéar JP. Twenty years' experience in the treatment of acoustic neuromas with fractionated radiotherapy: A review of 45 cases. Int J Radiat Oncol Biol Phys 2006;66:170-78.

31. Gonzalez LF, L ekovic GP, Eschbacher J, Coons S, Spetzler RF. $A$ true malignant schwannoma of the eighth cranial nerve: $A$ case report. Neurosurgery 2007;6:E421-22.

32. $\mathrm{M} \mathrm{clver} \mathrm{JI,} \mathrm{Pollock} \mathrm{BE.} \mathrm{Radiation-induced} \mathrm{tumor} \mathrm{after}$ stereotactic radiosurgery and whole brain radiotherapy: Case report and literature review. J N eurooncol 2004;66:301-05.

33. V ogel JJ, Godefroy WP, van der M ey A G, le Cessie S, $K$ aptein AA. Illness perceptions, coping and quality of life in vestibular schwannoma patients at diagnosis. O tol Neurotol 2008;29:839-45.

34. Parving A, Tos M, Thomsen J, M øller H, Buchwald C. Some aspects of life quality after surgery for acoustic neuroma. A rch Otolaryngol Head Neck Surg 1992;118:1061-64.
35. Gouveris HT, M ann WJ. Quality of life in sporadic vestibular schwannoma: A review. ORL J Otorhinolaryngol Relat Spec 2010;72:69-74.

36. Whitmore RG, U rban C, Church E, Ruckenstein M, Stein SC, Lee JY. Decision analysis of treatment options for vestibular schwannoma. J Neurosurg 2011;114:400-13.

37. Cheung SW, A randa D, D riscoll CL, Parsa A T. M apping clinical outcomes expectations to treatment decisions: An application to vestibular schwannoma management. Otol Neurotol 2010; 31:284-93.

38. Banerjee R, M oriarty JP, Foote RL, Pollock BE. Comparison of the surgical and follow-up costs associated with microsurgical resection and stereotactic radiosurgery for vestibular schwannoma. J Neurosurg 2008;108:1220-24.

39. V erma S, A nthony R, T sai V, Taplin M, Rutka J. Evaluation of cost effectiveness for conservative and active management strategies for acoustic neuroma. Clinical Otolaryngology 2009; 34:438-46.

40. Telian SA. M anagement of the small acoustic neuroma: A decision analysis. A m J Otol 1994;15:358-65.

41. M orrison D. M anagement of patients with acoustic neuromas: A M arkov decision analysis. L aryngoscope 2010;120:783-90.

42. Committee on Hearing and Equilibrium guidelines for the evaluation of hearing preservation in acoustic neuroma (vestibular schwannoma). A merican A cademy of Otolaryngology-H ead and N eck Surgery Foundation, Inc. Otolaryngol Head Neck Surg 1995;113:179-80. 